\title{
Strategic Capacity Investment under Hold-up Threats: The Role of Contract Length and Width*
}

\author{
Laure Durand-Viel ${ }^{\dagger} \quad$ Bertrand Villeneuve ${ }^{\ddagger}$
}

May 28, 2013

\begin{abstract}
This article analyzes the impact of the length of incomplete contracts on investment and surplus sharing. In the bilateral relationship explored, the seller controls the input and the buyer invests. With two-part tariffs, the length of the contract is irrelevant: surplus is maximal and goes all to the seller. If total surplus is targeted, or if sellers are left free, contracts will be as short as possible. If regulators can favor buyers, prohibibiting nonlinear pricing and forcing long contracts are the best solutions. The interaction of a restriction (width) with another (length) is explained in detail. Pipelines and large industrial appliances are the main examples.
\end{abstract}

JEL Codes: D42, D45, D92, L95.

Keywords: Long-term Contracts, Incomplete Contracting, Investment.

\section{Introduction}

Some equipment is very long-lived, and yet its size is determined once for all and cannot be readjusted. When the investor makes such a long-term decision about capacity, he needs to anticipate the possible evolution of the prices of equipment-related inputs and outputs. He will often seek to obtain guarantees about future prices, but the equipment might last longer than any reasonable contract duration. This might be a concern when the equipment uses an input produced by a firm with market power. If commitment is limited, investors may fear hold-up, i.e. unilateral price increases that will extract rent from the installed capital (Williamson, 1971). The energy sector at large offers a number of instances in which trade-offs must be found via contracts.

The paper examines investments for which the installed capacity will act as a cap on subsequent purchases. Once the investment has been made, buyers are locked into the chosen technology or standard, but they keep the flexibility to purchase less than nominal

\footnotetext{
*This work was supported by FiME (laboratoire de Finance des Marchés de l'Energie) and the "Chaire Finance et Développement Durable - Approches Quantitatives".

†Autorité de la Concurrence and Université Paris-Dauphine.

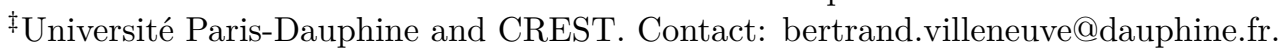


capacity. The obvious examples are oil and gas pipelines, and most large industrial fuelconsuming appliances. Ex post, elasticity in production is asymmetric: it is easier to scale down production than to overpass nominal capacity.

Equilibrium strategies of firms trying to attract customers who, once locked in by their investment choice, will be at the mercy of their market power recall the industrial organization literature on standard competition or aftermarket monopolization (Shapiro, 1995, Chen et al., 1998, Reitzes and Woroch, 2008). These models make rather extreme assumptions about the link between equipment and complementary goods (either strict complementarity: one aftermarket good for one primary good, see Carlton and Waldman, 2010, or independence: demand ex post can be adjusted freely, e.g. Borenstein et al., 2000, Morita and Waldman, 2004). In our model, complementarity is asymmetric: the buyer cannot consume more than his capacity allows, but he is free to consume less. The capacity constraint is carefully chosen by the buyer: investment itself, as well as the threat of scaling down demand anytime, is a strategic tool against the seller's potential abuses. In effect, the seller must not only convince the buyer to engage in a specific relationship with him, but he must incite him to make a sufficiently large investment in capacity and to use it: accordingly, he must guarantee reasonable tariffs if he doesn't want to kill the goose that lays golden eggs.

In a perfect world, it would be possible to sign a complete contract that would give adequate incentives to make the optimal investment. Sometimes, well designed incomplete contracts are also efficient (Crawford, 1988, Rey and Salanié, 1990, for the efficiency of repeated short contracts, or Guriev and Kvasov, 2005, for continuously renegotiated finite term contracts). The contracts with limited width that we study in this paper are optimized with respect to length in compensation. Conventional wisdom has it that long contracts protect investors (special interest) as well as investment (general interest). Eventually, one would expect the investment level and social welfare to rise as the commitment duration increases, the extra surplus being shared between the parties depending on their bargaining powers. This is the thesis in the empirical study of coal contracts by Joskow (1987). He finds evidence of a positive relationship between asset "specificity" - as measured by different proxies - and contract length (see also Neumann and Hirschhausen, 2008). On the other hand, caution dictates that incomplete contracts should not last too long because they could become very inefficient. See also Creti and Villeneuve (2004) for a survey on take-or pay gas contracts. A time limit may be necessary to avoid applying obsolete clauses, especially if some random factor drives the economy away from the initial conditions on the basis of which the contract was calibrated (Crocker and Masten, 1988, for natural gas contracts). Still, the trade-off is not expected to lead to very short contracts as the benefits of commitment are still there.

Our model shows a simple and realistic context in which the shortest contract is the best. Contracts are so conceived that the commitment period concentrates the incentives whereas the rest of the time is for rent extraction. When the commitment period is long, the committed price chosen pursues both objectives, since rent extraction cannot be differed without opportunity cost. Since these objectives are antagonistic, a longer contract is less efficient at inducing investment. The negative impact on social welfare of a longer contract 
is very unequally shared between the parties: the seller is a net loser, whereas the buyer produces less surplus but he is still a net winner as he can keep a much larger part of it. ${ }^{1} \mathrm{~A}$ longer contract actually protects expropriable investors rather than the investment itself.

We also argue that simpler contracts, such as contracts with purely linear prices, even though less efficient in general, are robust and, more importantly, that they guarantee buyers a share of the surplus. Linear pricing as well as the duration of the contract could be seen as general rules that parties can agree on beforehand to prevent contract failure, or that can be imposed by regulators in the interest of buyers, while letting the parties negotiate about contractual details. In our view, contract length and width (price structure) can be viewed as key features of the "constitution' governing the ongoing relationship" (Goldberg, 1976, p. 428). This view motivates our comparative statics analysis of contract length in relation with restrictions on the price structure.

The paper is organized as follows. Section 2 sets up the model. We analyze various scenarios with two-part tariffs in section 3. Linear contracts are justified and characterized in section 4. The proofs are relegated to the Appendix.

\section{General setting}

\subsection{Trade and payoffs}

The game involves two players: the seller and the buyer. The model is in continuous time with infinite horizon. At instant $t$, the seller sells to the buyer a quantity $q_{t}$ of a commodity produced at a constant unit $\operatorname{cost} c$; he receives in exchange a payment $\tau_{t}$, expressed in units of the numéraire good.

The instantaneous utility of the buyer is quasi-linear. It can be written (up to some irrelevant constant) $u\left(q_{t}\right)-\tau_{t}$. We will use the iso-elastic utility function

$$
u(q)=\frac{\varepsilon d^{\frac{1}{\varepsilon}}}{\varepsilon-1} q^{\frac{\varepsilon-1}{\varepsilon}}
$$

where $d$ is a positive scale parameter and where $\varepsilon$ is the price elasticity of demand. We assume that $\varepsilon>1$.

The seller sets the tariffs $\left\{\tau_{t}(\cdot), t \geq 0\right\}$ where the argument of $\tau_{t}(\cdot)$ is $q_{t}$. Only linear and two-part tariffs are used. The general shape of the total payment at date $t$ to the seller for quantity $q_{t}$ is therefore $\tau_{t}\left(q_{t}\right)=m_{t}+p_{t} q_{t}$, where $m_{t}$ is a per-time-unit fixed fee and $p_{t}$ is the marginal price.

Thus, the instantaneous buyer demand $Q(\cdot)$ exhibits constant price elasticity:

$$
Q\left(p_{t}\right)=d p_{t}^{-\varepsilon} .
$$

\footnotetext{
${ }^{1}$ Castaneda (2006) also finds a negative effect of longer contracts on investment, but in his model both parties are worse off. The contract in his analysis is such that the buyer pays upfront a lump sum and that the seller invests after acceptance. The buyer thus is reluctant to accept long contracts that would establish extortion for a long time, meaning in turn that the seller's return on them is poor, which depresses investment.
} 
The inverse demand function $Q^{-1}$ is denoted $P$.

The consumption of the commodity requires an equipment with an indefinite service life, installed at date $t=0$, whose size $A$ determines maximum consumption once and for all. For example if the equipment is a pipeline, imports cannot exceed the pipeline's capacity:

$$
q_{t}=\min \left\{Q\left(p_{t}\right), A\right\} .
$$

Unless otherwise specified, the investment cost ( $k$ per unit) is assumed to be borne by the buyer, who chooses non-cooperatively the investment size $A$ (the case where the seller is the investor is also discussed further on). The future is discounted at rate $r$.

The intertemporal surplus of the buyer is

$$
S=\int_{0}^{+\infty}\left(u\left(q_{t}\right)-\tau_{t}\left(q_{t}\right)\right) e^{-r t} d t-k A .
$$

The intertemporal profit of the seller is

$$
\Pi=\int_{0}^{+\infty}\left(\tau_{t}\left(q_{t}\right)-c q_{t}\right) e^{-r t} d t .
$$

The first best. The social optimum requires $u^{\prime}\left(q_{t}\right)=c+r k$, which means constant consumption: $\forall t, q_{t}=Q(c+r k)$. The term $r k$ is the unit amortization cost of the durable investment. The efficient investment is thus

$$
A^{*}=Q(c+r k) \text {. }
$$

\subsection{Market power and limited commitment}

Trade takes place only after the equipment has been built, however trading terms can be determined beforehand by means of an agreed tariff. $T$ is the commitment duration.

1. At date 0 , the seller makes a take-it-or-leave-it offer to the buyer consisting of tariffs $\left\{\tau_{t}(\cdot), t \in[0, T)\right\}$, valid until $T$. The buyer accepts this offer if it leaves him with a non-negative surplus.

2. The buyer invests $A$.

3. From $t=0$ to $T$, the buyer purchases $q_{t}$ at each date $t$.

4. At date $T$, the contract expires. At each date $t \geq T$, the seller makes a takeit-or-leave-it offer to the buyer consisting of tariff $\tau_{t}(\cdot)$. The buyer accepts this offer if it leaves him with a non-negative instantaneous surplus, and consumes the corresponding $q_{t}$. 
Without loss of generality, the seller's pricing strategies can be restricted to constant marginal prices over each period: $p_{0}$ during the first period $(0 \leq t<T)$ and $p_{T}$ during the second period (for $t \geq T$ ). The way the fixed payments are staggered over time within the first period does not matter in the sense that paying $m_{t}$ at all dates $t \in[0, T)$ is equivalent to an upfront payment ${ }^{2}$

$$
M_{0}=\int_{0}^{T} m_{t} e^{-r t} d t .
$$

The contract can be summarized by $\left(M_{0}, p_{0}\right)$.

In the $4^{\text {th }}$ step, the buyer cannot change his investment, thus whether the seller offers a durable contract or (as we assume) a short-term contract does not make a difference. Indeed, any optimal contract has to implement the same successive identical offers.

The following sections will discuss performance of the market with respect to the firstbest allocation. The analysis will focus on a bilateral relationship where the investor is the buyer; the case where the seller invests will also be discussed.

\section{Two-part tariffs}

\subsection{Unlimited commitment $(T=+\infty)$}

Implementation of the first-best is straightforward. If $p_{0}=c$ and the fixed fee is reasonable (i.e. such that the buyer participates), the buyer invests $A^{*}$. To extract the surplus, the seller must choose

$$
M_{0}=\frac{1}{r}\left(u\left(A^{*}\right)-(c+r k) A^{*}\right),
$$

which is the present value of the perpetual flow $u\left(A^{*}\right)-(c+r k) A^{*}$. This is the generalization of the well-known static result.

\subsection{Limited commitment $(0<T<+\infty)$}

At date $T$ the investment $A$ is sunk and nothing can prevent the seller from exerting holdup on the buyer by setting at each instant a tariff that captures the entire surplus from the relationship. For $t \geq T$, any tariff scheme $\left(m_{t}, p_{T}\right)$ such that $p_{T} \leq P(A)$ (to avoid under-consumption) and $m_{t}+\left(p_{T}-c\right) A=u(A)$ (to ensure participation) will do. A simple example is $p_{T}=c$ combined with a fixed fee $m_{t}=u(A), \forall t \geq T$.

The buyer anticipates that he will obtain no surplus from $T$ on. Assume the price during the first period is constant and denoted by $p_{0}$. After rearrangement, the buyer solves:

$$
\max _{A} \frac{1-e^{-r T}}{r}\left(u(A)-\left(p_{0}+\frac{r k}{1-e^{-r T}}\right) A\right)-M_{0},
$$

\footnotetext{
${ }^{2}$ Liquidity constraints on either player could limit this freedom, which we do not consider.
} 
where the expression reveals that, due to expropriation ex post, the investment $A$ has to be amortized during the first period. The equivalent flow cost of investment is thus $\frac{r k}{1-e^{-r T}}$ per unit of equipment.

The buyer will invest

$$
A=Q\left(p_{0}+\frac{r k}{1-e^{-r T}}\right)
$$

under the condition that $M_{0}$ is small enough to ensure participation.

The seller anticipates that he can obtain the total surplus from $T$ on, whose present value is $\frac{e^{-r T}}{r}(u(A)-c A)$. He solves

$$
\begin{aligned}
& \max _{M_{0}, p_{0}} \Pi\left(M_{0}, p_{0}\right)= M_{0}+\frac{1-e^{-r T}}{r}\left(p_{0}-c\right) Q\left(p_{0}+\frac{r k}{1-e^{-r T}}\right) \\
&+\frac{e^{-r T}}{r}\left[u\left(Q\left(p_{0}+\frac{r k}{1-e^{-r T}}\right)\right)-c Q\left(p_{0}+\frac{r k}{1-e^{-r T}}\right)\right] \\
& \text { s.t. } M_{0} \leq \frac{1-e^{-r T}}{r}\left[u\left(Q\left(p_{0}+\frac{r k}{1-e^{-r T}}\right)\right)-\left(p_{0}+\frac{r k}{1-e^{-r T}}\right) Q\left(p_{0}+\frac{r k}{1-e^{-r T}}\right)\right] .
\end{aligned}
$$

Clearly, the seller will choose the largest possible fixed fee and the corresponding profitmaximizing price.

Proposition 1. When the seller can commit to a two-part tariff $\left(M_{0}, p_{0}\right)$ for a limited duration $T$, he will set a marginal price below his marginal cost:

$$
p_{0}=c-\frac{e^{-r T}}{1-e^{-r T}} r k,
$$

This leads the buyer to undertake the optimal investment $A^{*}=Q(c+r k)$. The fixed fee allows the seller to capture the entire first-stage surplus:

$$
M_{0}=\frac{1-e^{-r T}}{r}\left(u\left(A^{*}\right)-(c+r k) A^{*}\right) .
$$

Then the following tariff allows him to capture the entire second-stage surplus:

$$
\text { For all } t>T, m_{t}=u\left(A^{*}\right) \text { and } p_{T}=c .
$$

An interesting feature of this game is that the consumption good is subsidized during the contract: it is priced below its marginal cost, the subsidy being the amortization of capital from date $T$ on, i.e. it is an advance compensation for the hold-up period. The shorter the contract duration and the larger the investment cost, the more generous the first-stage unit price reduction must be in order to stimulate investment. Remark that $p_{0}$ is negative if $T$ is sufficiently short.

The two instruments of the contract play different roles: $p_{0}$ is used to give the right investment incentives to the buyer, and $M_{0}$ transfers the surplus to the seller. The seller is willing to set a below-cost unit price in order to encourage investment because this does not prevent him from capturing the entire surplus from the relationship, through the fixed fee in the first stage, then by exerting hold-up after expiry of the contract. 


\subsection{No commitment}

Suppose now the seller cannot commit to a tariff $\left(M_{0}, p_{0}\right)$. This is the pure hold-up situation: once the investment is made, the seller will repeatedly set the same tariff $(m, p)$ that captures the entire ex post surplus for each period, which means that the buyer cannot recoup his investment costs. As a consequence, the buyer's surplus if he invests is negative. Therefore, there is no equilibrium with positive investment. No commitment and powerful tools is the worst-case scenario.

\subsection{Endogenous duration}

The first-best investment level can be attained whatever $T>0$. The interest of the alternative scenarios with respect to $T$ lies in the timing of transfers and the structure of tariffs. In all cases, the whole surplus goes into the seller's hands. In the analysis, the contract duration $T$ is treated as exogenous. Now suppose the seller or the buyer has the power to determine the contract's duration. All durations $T>0$ are equivalent for both players, while no commitment is inefficient, thus the choice will be any strictly positive duration.

\subsection{Investment by the seller}

Suppose the investment is made by the seller. Fixed fees allow a monopolistic seller to capture the entire surplus both during and after the contract, therefore it is optimal for him to choose the first-best investment level $A^{*}$ and to always set the variable price $p=c+r k$ to induce a consumption equal to $A^{*}$. In fact, whether the investment is undertaken by the seller or the buyer, two-part tariffs yield the efficient level of investment and allocate the surplus to the seller.

\section{Linear tariffs with expiry date}

Before studying the structure of the equilibrium with linear tariffs, we give two theoretical reasons why they are important in practice.

For the first argument, there is a basic result on two-part tariffs that must be recalled. Take the static standard monopoly in front a demand function. Suppose that the fixed part of a two-part tariff is capped by regulation, and let's see what happens when the cap goes down.

- As long as the cap is high enough, the constraint doesn't matter. The seller sets a marginal price equal to the marginal cost and extracts the entire surplus through the fixed fee. This is the best case for the seller and it is socially optimal.

- When the cap on the fixed part is binding and goes down, the seller inevitably decreases the fixed fee and raises the marginal price above marginal cost. The buyer's consumption decreases and his surplus stays at zero. 
- As the cap on the fixed part falls further down, the seller fixes the price at the standard monopoly level before being forced to a pure linear price. He continues to set the highest permissible fee.

The pure linear price is a limit case of the latter regime with the cap at zero. In that important regime, capped two-part tariffs all yield the same marginal price, quantity and investment level as linear tariffs. Only the surplus sharing is affected via the cap-dependent fixed fee (the buyer's surplus increases as the cap decreases). Therefore studying linear prices is not as restrictive as it may seem since the predictions encompass the case of two-part tariffs with relatively tight caps on fixed fees.

This being recalled, the first defense of linear pricing relies on its distributive role: it guarantees the buyer a share of the surplus. Linear pricing may emerge as a general rule for a wide set of contexts imposed in the buyer's interest. In the absence of information on future costs or preferences, it is extremely robust as a protection against full rent extraction.

Robustness of linear contracts is a property valuable to the seller for another reason. Consider $d$, the scale factor of demand (see equation 2). The optimal two-part tariffs all have marginal prices at marginal cost, but the fixed part is very sensitive to $d$. If information about $d$ is asymmetric, the first-best allocation cannot be implemented. The standard approach would be to search for an incentive-compatible menu of tariffs, with one tariff having a minimal fixed-fee. The exact structure of the menu highly depends on the details. In case the information cannot be probabilized and the support of $d$ contains small values, the robustness view has practical interest. We show indeed in what follows that the linear prices the seller should use are robust to asymmetric information about $d$ in the sense that linear tariffs do not depend on $d$ at equilibrium. This justifies ex post our interest for linear prices.

\subsection{A general characterization}

We assume now that, at all dates $t$, the buyer has to pay a linear price $p_{t}$, and that prices are guaranteed until date $T>0$ only.

A priori, during the first stage $t \in[0, T]$, different prices could be used at different dates. In fact, the following lemma proves that this is not necessary:

Lemma 1. If the seller uses linear prices at each date, it is always optimal for him to restrict himself to a constant price during the commitment period.

In the first stage (from $t=0$ to $T$ ) the price is $p_{0}$ and consumption is $q_{0}$, while in the second stage (from $t=T$ to $+\infty$ ) the price is $p_{T}$ and consumption is $q_{T}$. The game is sequential, with successive decisions $p_{0}, A, q_{0}, p_{T}, q_{T}$. As usual, it will be solved by backward induction, but a second lemma will eliminate further large classes of dominated strategies.

The buyer can either passively invest the capacity corresponding to the monopoly quantity, or use his investment choice to influence the outcome. In addition, the seller can either passively set $p_{0}$ at the anticipated marginal willingness to pay of the buyer, or use $p_{0}$ strategically to influence his investment behavior, typically to stimulate investment. 
This defines the following alternative behaviors. We shall see what they imply and which prevail in equilibrium.

Definition 1. The buyer is said to be active when his investment choice A induces a response $p_{T}$ from the seller that differs from the unconstrained monopoly price $\frac{\varepsilon c}{\varepsilon-1}$. Otherwise he is said to be passive.

Definition 2. The seller is said to be active when his price choice $p_{0}$ induces a response A from the buyer that differs from $A=Q\left(p_{0}\right)$. Otherwise he is said to be passive.

To begin with, we will prove that the investment is never oversized in the second stage, and that the second-stage price equates demand with capacity. Otherwise, the seller could slightly adjust his first-period price without affecting the second period; setting the price $p_{0}$ closer to the unconstrained monopoly price would enhance profits. The next lemma also proves that the investment can never be oversized in the first stage either.

\section{Lemma 2.}

1. When the buyer is active, $p_{T}=P(A)>\frac{\varepsilon c}{\varepsilon-1}$.

2. When the buyer is passive, $p_{0}=p_{T}=P(A)=\frac{\varepsilon c}{\varepsilon-1}$, and the seller is also passive.

3. At equilibrium, $A \leq Q\left(p_{0}\right)$.

The lemmas enable us to summarize the four candidate regimes in Table 1 .

\begin{tabular}{|c|c|c|c|c|}
\hline & \multicolumn{4}{|c|}{ Seller } \\
\hline & & Active & & Passive \\
\hline \multirow[b]{4}{*}{. } & & $<p_{T}$ & $p_{0}=$ & $p_{T}$ \\
\hline & $p_{T}$ & $>\quad \frac{\varepsilon c}{\varepsilon-1}$ & $p_{T}>$ & $\frac{\varepsilon c}{\varepsilon-1}$ \\
\hline & $A$ & $=Q\left(p_{T}\right)<Q\left(p_{0}\right)$ & $A=$ & $Q\left(p_{0}\right)=Q\left(p_{T}\right)$ \\
\hline & & IMPOSSIBLE & $\begin{aligned} p_{0} & = \\
p_{T} & = \\
A & =\end{aligned}$ & $\begin{array}{l}p_{T} \\
\frac{\varepsilon c}{\varepsilon-1} \\
Q\left(\frac{\varepsilon c}{\varepsilon-1}\right)\end{array}$ \\
\hline
\end{tabular}

Table 1: The 4 types of equilibrium.

Theorem 1 (Equilibrium prices in the general case).

(a) If $\frac{c}{r k} \geq(\varepsilon-1) e^{r T}$, both parties are passive and

$$
p_{0}=p_{T}=\frac{\varepsilon c}{\varepsilon-1} .
$$

(b) If $(\varepsilon-1) e^{r T}-\frac{1}{\varepsilon} \leq \frac{c}{r k}<(\varepsilon-1) e^{r T}$, the buyer is active and the seller is passive, and

$$
p_{0}=p_{T}=r e^{r T} \varepsilon k .
$$


(C) If $\frac{c}{r k}<(\varepsilon-1) e^{r T}-\frac{1}{\varepsilon}$, both parties are active and

$$
\left\{\begin{array}{l}
p_{0}=\frac{1}{1-e^{-r T}}\left[\left(1-\frac{\varepsilon e^{-r T}}{\varepsilon+e^{-r T}}\right) \frac{\varepsilon c}{\varepsilon-1}+\left(1-\frac{\varepsilon^{2} e^{-r T}}{\varepsilon+e^{-r T}}\right) \frac{r k}{\varepsilon-1}\right] \\
p_{T}=\frac{\varepsilon^{2}(c+r k)}{\left(\varepsilon+e^{-r T}\right)(\varepsilon-1)}
\end{array}\right.
$$

Since we are mostly interested in the situation where both players use their opportunities of strategically influencing the other party's behavior, we will concentrate the analysis in the text on the case where the seller and the buyer are active. For the other cases, Figure 1 illustrates how changing $T$ can make the equilibrium prices cross all three regimes (details are given in subsection A.5).

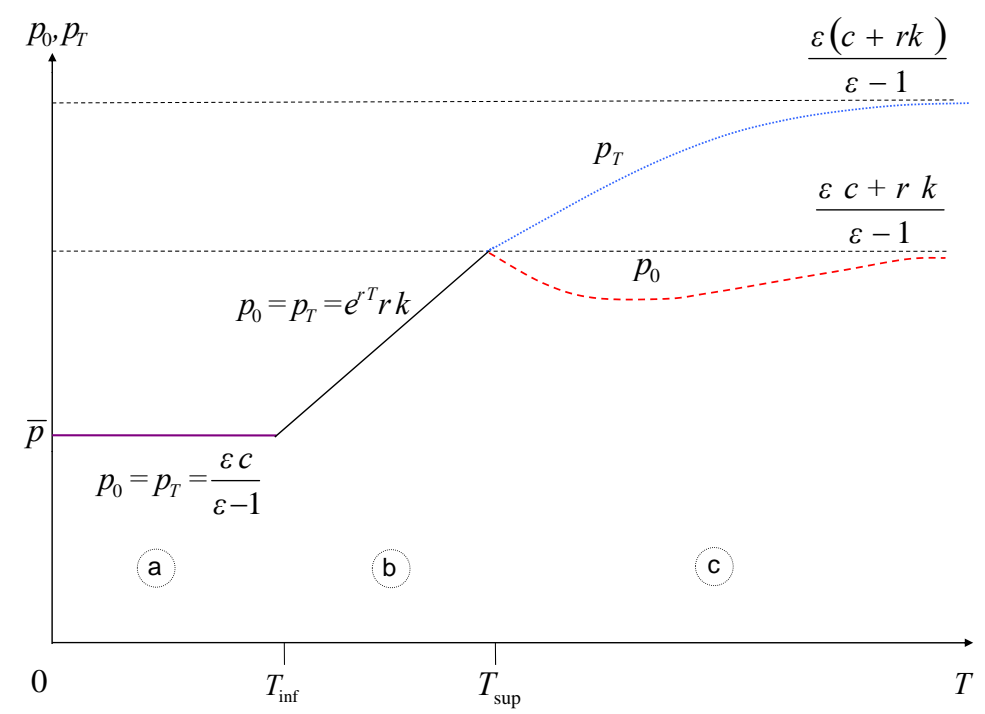

Figure 1: Equilibrium prices as a function of the contract duration (when $\frac{c}{r k}>\varepsilon-1$ ).

From the theorem, we see that a necessary and sufficient condition for both players to be active for all $T$ is

$$
\frac{c}{r k}<\varepsilon-1-\frac{1}{\varepsilon}
$$

Note that for the right hand-side to be positive, the elasticity of demand must exceed $\frac{1+\sqrt{5}}{2}$, the Golden Ratio ( $\simeq 1.6$ ). Equilibrium prices as a function of $T$ are given in Figure 2.

\subsection{The value of commitment}

One could think that longer commitment periods are necessarily better for the seller: any price profile that can be implemented with a short commitment duration (say $T_{1}$ ) can be 


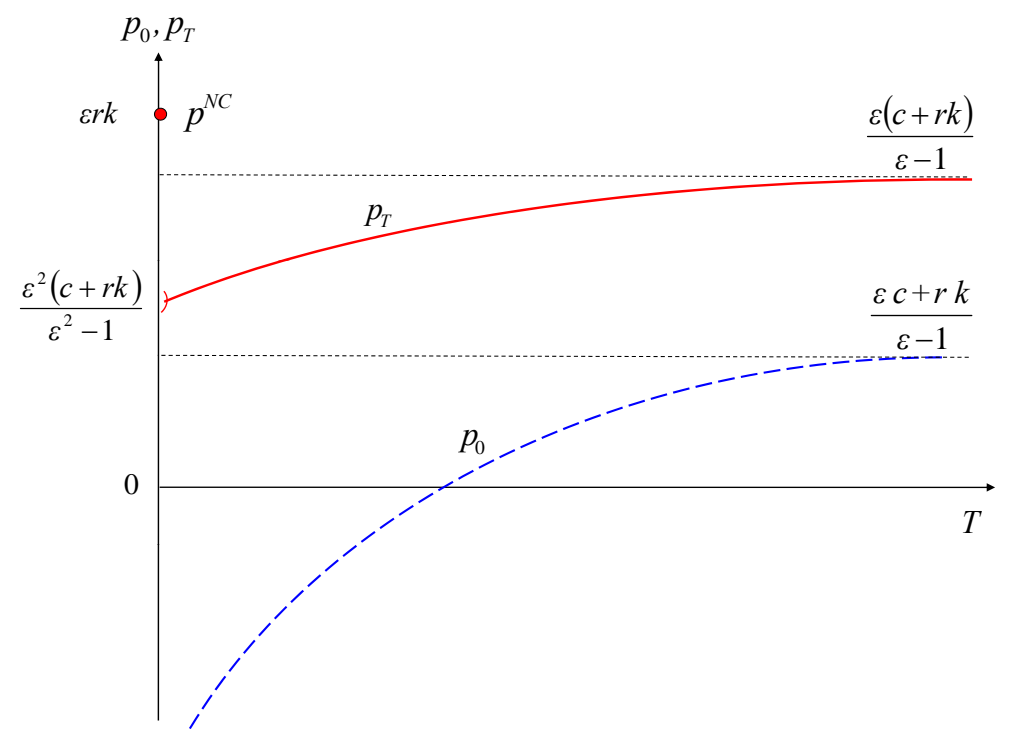

Figure 2: Equilibrium prices as a function of the contract duration (when $\frac{c}{r k}<\varepsilon-1-\frac{1}{\varepsilon}$ ). $N C$ corresponds to the no-commitment scenario (subsection 4.3).

implemented with a longer commitment duration (say $T_{2}$ ). But from this, it doesn't follow that the (so to speak) $T_{2}$-buyer will replicate the behavior of the (so to speak) $T_{1}$-buyer.

Assume that the $T_{1}$-buyer and the $T_{2}$-buyer face the same price profile and that they consider investing $A$. How do they value a marginal increase in $A$ ? Identically from $t=0$ to $t=T_{1}$ : price is fixed anyway. Identically also from $t=T_{2}$ on: the post-commitment price is a function of capacity only. Now, an increase in capacity will yield a price reduction between $T_{1}$ and $T_{2}$ for the $T_{1}$-buyer, whereas the price remains constant in that interval for the $T_{2}$-buyer. This means that the value of increasing $A$ is necessarily less for the $T_{2}$-buyer than for the $T_{1}$-buyer. There is no ambiguity: facing the same price profile, the $T_{2}$-buyer (whose contract is longer) invests less than the $T_{1}$-buyer. This shows that a mere replication doesn't work.

Since $A=Q\left(p_{T}\right)$ is a decreasing function of $p_{T}$, which (from Theorem 1) is an increasing function of $T$ in all regimes, the following paradoxical result obtains.

Proposition 2 (Investment). The equilibrium investment level decreases with respect to the contract duration for all $T>0$.

Since the seller cannot commit to refrain from extracting rent after contract expiry, his only means to stimulate investment is to offer at $t=0$, before $A$ is chosen, the guarantee of a low price until $T$. The smaller $T$, the more generous the bargain must be: $p_{0}$ can even be negative, i.e. consumption is subsidized during the contract when the contract duration is short. For example, when $T$ is close to zero but still strictly positive, $p_{0}$ becomes infinitely negative in the case where $\frac{c}{r k}<\varepsilon-1-\frac{1}{\varepsilon}$ (see Figure 2). 
Remark. Intuitively, one would think that the equilibrium prices are increasing functions of the investment cost, since a higher $k$ tends to dampen investment and consumption. This is true for the hold-up price $p_{T}$, but not necessarily for the contract price: it may be worth the seller's while to set a low $p_{0}$, thus sacrificing first-stage profits, to induce a higher investment and more second-stage sales at the hold-up price $p_{T}=P(A)$. See the analysis in subsection A.6 and Figure 4 therein.

Since social welfare increases with respect to the investment level, Proposition 2 implies that welfare decreases with respect to the contract duration. As for the seller's profit and the buyer's surplus, when $\frac{c}{r k}<\varepsilon-1-\frac{1}{\varepsilon}$, they read respectively

$$
\begin{aligned}
\Pi & =\frac{1}{r} \frac{c+r k}{\varepsilon-1} Q\left(\frac{\varepsilon^{2}(c+r k)}{\left(e^{-r T}+\varepsilon\right)(\varepsilon-1)}\right) \\
S & =\frac{1}{r} \frac{1-e^{-r T}+\frac{1}{\varepsilon} e^{-r T}}{\varepsilon-1} \frac{\varepsilon^{2}(c+r k)}{\left(e^{-r T}+\varepsilon\right)(\varepsilon-1)} Q\left(\frac{\varepsilon^{2}(c+r k)}{\left(e^{-r T}+\varepsilon\right)(\varepsilon-1)}\right) .
\end{aligned}
$$

Proposition 3 (Welfare). When $\frac{c}{r k}<\varepsilon-1-\frac{1}{\varepsilon}$, for all $T>0$,

1. Social welfare decreases with respect to $T$.

2. The seller's profit decreases with respect to $T$.

3. The buyer's surplus increases with respect to $T$.

See Figure 3.

With linear prices, more commitment through longer contracts is detrimental to the seller and has the effect of depressing investment. In a nutshell, the first price $p_{0}$ serves to push investment whereas $p_{T}$ serves to extract rents: the two successive prices approach a two-part tariff, the first price providing the marginal incentives (investment) and the second price the inframarginal ones (participation). Though the performance of these linear prices is imperfect, this view clarifies the effects of $T$ across agents, and for the economy, as we explain now.

At the beginning of Section 4, we recalled how a cap on the fixed fee benefits the buyer. Longer durations are similar to lower (i.e. more advantageous) caps: the longer the commitment, the smaller the impact of the hold-up period is. In compensation, as the second price loses impact when $T$ increases, the seller has to mix in the first price two objectives (incentives and rent extraction), which is the typical inefficiency of plain static linear prices. What about the buyer? As $T$ increases, the buyer's surplus increases even though the surplus to be shared is smaller.

\subsection{No commitment}

An alternative scenario which doesn't fit in the previous structure, has to be considered: no commitment at all. The timing is simplified:

1. The buyer invests $A$ at a unit cost $k$. 


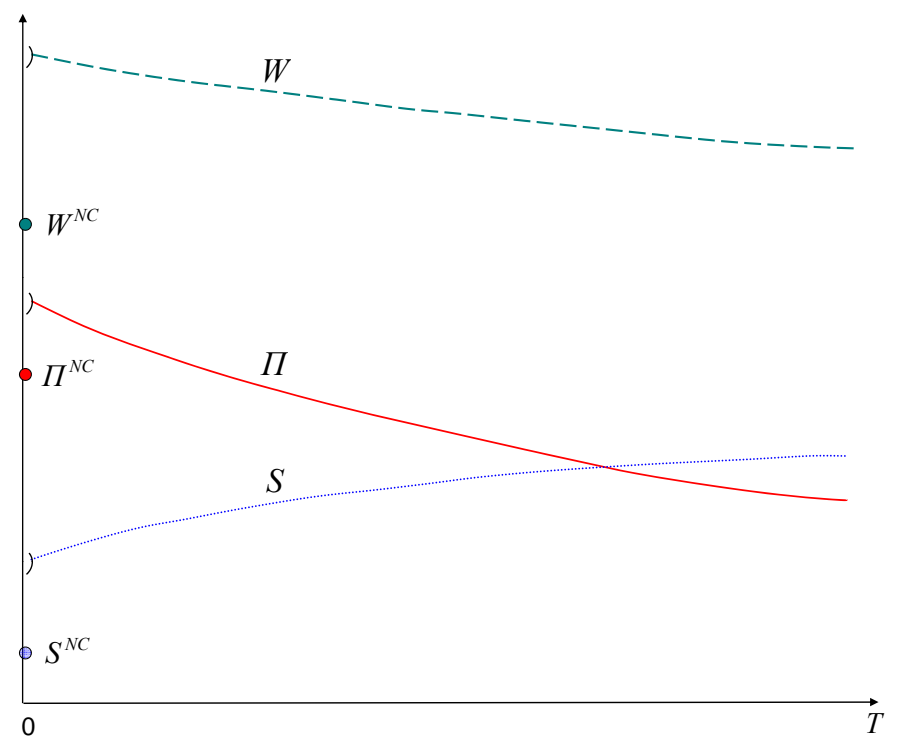

Figure 3: Profit of the seller, surplus of the buyer and social welfare as a function of the contract duration (when $\frac{c}{r k}<\varepsilon-1-\frac{1}{\varepsilon}$ ). $N C$ corresponds to the no-commitment scenario (subsection 4.3).

2. At each date $t$, the seller makes a take-it-or-leave-it offer $p_{t}$ to the buyer. If the buyer accepts, he buys $q_{t}$.

In effect, this case differs, as we shall see, from the case $T \rightarrow 0$ in the previous game. Indeed, whenever $T>0$, and whatever small $T$ may be, there are a price before and a price after $A$ is chosen.

Clearly the same price and quantities (denoted respectively by $p$ and $q$ ) will be chosen at all dates. The buyer anticipates that he will suffer hold-up as soon as the investment is realized, but extortion is imperfect as linear prices are used.

The following proposition summarizes the results.

Proposition 4 (No commitment at all). The equilibrium price and investment level are

$$
\begin{aligned}
p & =\max \left\{\frac{\varepsilon c}{\varepsilon-1}, \varepsilon r k\right\}, \\
A & =\min \left\{Q\left(\frac{\varepsilon c}{\varepsilon-1}\right), Q(\varepsilon r k)\right\} .
\end{aligned}
$$

Since in both cases the capacity is fully used $(q=A=Q(p))$, the outcome is ex post efficient; however, ex ante efficiency is not achieved. Indeed, the equilibrium price is always higher than the social optimum $p=c+r k$, which implies that the equilibrium investment level is always suboptimal.

No commitment and "some" commitment $(T>0)$ are qualitatively different. In other terms, to encourage investment, the smallest contract is the best (Proposition 2), no con- 
tract at all is the worst case. See $N C$ (no commitment) prices, profits and welfare in Figures 2 and 3 . In particular, note that $p^{N C}$ is larger than any $p_{0}$ or $p_{T}$.

\subsection{Investment by the seller}

An alternative assumption could be that the investment is undertaken by the seller. Let us examine the following game:

1. The seller chooses the investment size $A$ and sets price $p$.

2. The buyer purchases $q$ indefinitely.

Commitment is not relevant here, because the optimal price for the seller does not change over time. There is no need to create incentives and extract rents in two separate episodes. This means that contract duration does not matter.

The seller solves the program of a monopolist whose marginal cost is $c+r k$ : the equilibrium is characterized by

$$
\begin{aligned}
p & =\frac{\varepsilon(c+r k)}{\varepsilon-1}, \\
A & =Q\left(\frac{\varepsilon(c+r k)}{\varepsilon-1}\right) .
\end{aligned}
$$

This game is essentially equivalent to the previous game with $T=+\infty$ with buyer investment: the outcomes (investment level, profit and surplus of the parties) are the same. The seller simply increases the commodity price by $r k$ to pass on his investment cost.

Does this solve the seller's problem? In fact, the seller would prefer to let the buyer invest. The reasoning is as follows. If the seller invests, he can invest at the level that he wishes, but the linear prices limit his ability to extract the rent thus generated. Since the investment cost is difficult to recoup, his incentive to invest in the first place is limited. Conversely, if the buyer invests, the linear prices protect his effort (he is able to keep a fraction of the surplus generated). So the incentives that the seller gives to the buyer are leveraged by the buyer's own incentives, which makes the seller's contribution to investment (through rebates) relatively small. This allows the seller to extract a bigger rent through the second price $p_{T}$.

\section{Conclusion}

In this paper, we have examined a situation where contracts are incomplete in several respects: both the pricing structure and the duration of commitment are restricted. All the pricing schemes that we have analyzed share the following feature: some commitment is always better than none. The reason is intuitive: the seller can offer a significant rebate that gives the buyer perfect or at least fairly good incentives to invest. The commitment period secures the indispensable thrust. 
A longer contract never gives higher investment incentives. Tariffs with a sufficient "width" (such as two-part tariffs) are powerful enough to make the contract length irrelevant. With linear tariffs, the investment level is a strictly decreasing function of the contract length. A shorter subsidy period concentrates the effort, but it doesn't diminish the incentives that can be conveyed. Moreover, a shorter commitment limits the postponement of the profitable hold-up period, mechanically limiting the opportunity cost for the seller. Accordingly, when the contract duration is very short, the seller will choose to subsidize investment heavily to maximize the surplus that will subsequently be extracted. In this sense, length and width of the contract are complements.

With linear tariffs, social welfare, the seller's profit and the buyer's surplus do not vary in parallel when contract length changes. Contrary to the seller's profit and social welfare, the buyer's surplus increases with length. To see why we have these results, an analogy with the static monopoly model is useful. Indeed, in that classical case, it is possible to increase the buyer's surplus by restricting the use of two-part tariffs. One option is to put a cap on the fixed part, or even to eliminate it. Albeit inefficient, pure linear pricing may afford the buyer more surplus than other solutions. In our model, the first period is the one during which investment incentives are set up by the seller. The seller tries to make the buyer invest and consume as if prices were exactly equal to marginal costs. The second period in contrast acts like the fixed part, serving to extract surplus: requiring that the commitment period be long means that there is a cap on the rent extracted by the seller, which benefits the buyer.

The analogy is imperfect, if only because two linear prices cannot strictly replicate nonlinear tariffs. Nevertheless, it simply shows that a rule forcing linear prices and longer commitment on prices could be set up as a "universal" means to protect buyers against sellers' market power. This would be at the expense of investment.

Long-term contracts on specific assets have been repeatedly under the scrutiny of the competition authorities in Europe. The divide is between the pro-competition line of argument (foreclosure establishes durably abusive market power, e.g. Aghion and Bolton, 1987 ) and the pro-investment line of argument (contracts offer protection against expropriation). The 2007 Energy Sector Inquiry states that "long-term supply agreements seem to foreclose the availability of crucial inputs for actual or potential competition" (p. 66). On the other hand, the 2004 Directive on Security of Natural Gas Supply emphasizes their positive impact on investment, underlining that "long-term contracts have played a very important role in securing gas supplies for Europe and will continue to do so" (recital 11). Though the latter view is largely supported by the energy industry, the link between contract duration and investment incentives in a noncompetitive environment has not been thoroughly established by economic theory. One reason is that duration is not just another parameter in a contract, complete or incomplete. Its interaction with other dimensions can be quite counterintuitive. 


\section{References}

Aghion, Philippe and Patrick Bolton (1987): "Contracts as a Barrier to Entry", American Economic Review, 77(3), 388-401.

Borenstein, Severin, Jeff Mackie-Mason and Janet Netz (2000): "Exerting Market Power in Proprietary Aftermarkets", Journal of Economics and Management Strategy, 9(2), 157-88.

Carlton, Dennis and Michael Waldman (2010): "Competition, Monopoly, and Aftermarkets", Journal of Law, Economics \& Organization, 26, 54-91.

Castaneda, Marco A. (2006): "The Hold-up Problem in a Repeated Relationship", International Journal of Industrial Organization, 24(5), 953-970.

Chen, Zhiqi, Thomas Wayne Ross and William T. Stanbury (1998): "Refusals to Deal and Aftermarkets", Review of Industrial Organization, 13, 131-151.

Council of the European Union (2004): Council Directive 2004/67/EC of 26 April 2004 concerning measures to safeguard security of natural gas supply.

Crawford, Vincent P. (1988): "Long-Term Relationships Governed by Short-Term Contracts", American Economic Review, 78, 485-499.

Creti, Anna and Bertrand Villeneuve, Bertrand (2004): "Long-term Contracts and Take-or-pay Clauses in Natural Gas Markets", Energy Studies Review, 13, 75-94.

Crocker, Keith J. and Scott E. Masten (1988): "Mitigating Contractual Hazards: Unilateral Options and Contract Length", RAND Journal of Economics, 19(3), 327-343.

European Commission (2007): DG Competition report on energy sector inquiry, SEC (2006) 1724, 10 January 2007.

Goldberg, Victor P. (1976): "Regulation and Administered Contracts", Bell Journal of Economics, 7(2), 426-448.

Guriev, Sergei and Dmitriy Kvasov (2005): "Contracting on Time", American Economic Review, 95, 1369-1385.

Joskow, Paul L. (1987): "Contract Duration and Relationship-Specific Investments: Empirical Evidence from Coal Markets", American Economic Review, 77(1), 168-185.

MacLeod, W. Bentley and James M. Malcomson (1993): "Investments, Holdup, and the Form of Market Contracts", American Economic Review, 83, 811-837.

Morita, Hodaka and Michael Waldman (2004): "Durable Goods, Monopoly Maintenance, and Time Inconsistency", Journal of Economics and Management Strategy, 13(2), 273-302.

Neumann, Anne and Christian von Hirschhausen (2008): "Long-Term Contracts and Asset Specificity Revisited: An Empirical Analysis of Producer-Importer Relations in the Natural Gas Industry", Review of Industrial Organization, 32(2), 131-143.

Reitzes, James D. and Glenn A. Woroch (2008): "Competition for Exclusive Customers: Comparing Equilibrium and Welfare Under One-Part and Two-Part Pricing", Canadian Journal of Economics/Revue canadienne d'économique, 41(3), 1046-1086.

Rey, Patrick and Bernard Salanié (1990): "Long-term, Short-term and Renegotiation: On the Value of Commitment in Contracting", Econometrica, 58, 597-619.

Shapiro, Carl (1995): "Aftermarkets and Consumer Welfare: Making Sense of Kodak", Antitrust Law Journal, 63, 483-511.

Williamson, Oliver E. (1971): "The Vertical Integration of Production: Market Failure Considerations", American Economic Review, Papers and Proceedings, 61(2), 112-123. 


\section{A Appendix}

\section{A.1 Proof of Proposition 1}

Proof in the text.

\section{A.2 Proof of Lemma 1}

Consider a contract of length $T$, such that the producer commits to a price profile $\left\{p_{t}\right\}_{0 \leq t \leq T}$. We assume that for all real number $x$, sets of dates like $\left\{t \mid p_{t} \leq x\right\}$ are Lebesgue-measurable (a measure denoted by $d$ ), which is not restrictive in our context and it is technically more rigorous for the argument. The reasoning will be developed in three steps.

Step 1. First we shall prove that at equilibrium $A \leq Q\left(\frac{\varepsilon c}{\varepsilon-1}\right)$. Suppose instead that $A>Q\left(\frac{\varepsilon c}{\varepsilon-1}\right)$ : then the post-commitment price is $p_{T}=\frac{\varepsilon c}{\varepsilon-1}$, and $A$ is oversized in the period following contract expiry. We denote by $I$ the measurable set of dates in $[0, T]$ for which $p_{t} \leq p(A)$ (i.e. those dates for which consumption equals $A$ ).

(i) All prices above $p(A)$ (i.e. for $t \in[0, T] \backslash I)$ can be changed with profit to $\frac{\varepsilon c}{\varepsilon-1}$ : some increase and the others decrease, depending on their position relative to $\frac{\varepsilon c}{\varepsilon-1}$. These changes have no effect on the choice of $A$, consumption at those dates being unconstrained by $A$ before and after the change for all $t \in[0, T] \backslash I$. If $d(I)=0$, then we have shown that the price schedule was not optimal. If $d(I)>0$, we have to proceed with the proof.

(ii) We denote the "weight" of $I$ by $\mu(I)=\int_{I} e^{-r t} d t$. Prices in $I$ can be replaced by the weighted mean

$$
p_{I}=\frac{\int_{I} p_{t} e^{-r t} d t}{\mu(I)},
$$

without changing the seller's profits or the buyer's investment. Indeed, $p_{I}$ is in the convex hull of $\left\{p_{t} \mid t \in I\right\}$, and thus verifies $Q\left(p_{I}\right) \geq A$ : the constraint is still binding.

(iii) We have established in (i) and (ii) that any price profile can be replaced (with profit) with another price profile where price is a constant $\frac{\varepsilon c}{\varepsilon-1}$ over $[0, T] \backslash I$ and a constant $p_{I}<\frac{\varepsilon c}{\varepsilon-1}$ over $I$.

The buyer will equate $q_{t}$ with $A$ for $t \in I$ only, and will be strictly unconstrained at other dates, so that his program can be rewritten, for the only part that depends on $A$ :

$$
\max _{A} \mu(I) \cdot\left[u(A)-\left(p_{I}+\frac{k}{\mu(I)}\right) A\right] .
$$

At equilibrium the buyer's best choice is

$$
q_{t}=A=Q\left(p_{I}+\frac{k}{\mu(I)}\right) .
$$

If he anticipates this reaction, the seller wants to approach as much as possible the interior solution of the corresponding profit maximizing program. This interior solution is

$$
\frac{\varepsilon c+\frac{k}{\mu(I)}}{\varepsilon-1}>\frac{\varepsilon c}{\varepsilon-1} \text {. }
$$


In any case, the initial pricing choice appears not to be optimal. This proves step 1 by contradiction.

Step 2. Then we prove that consumption cannot be lower than chosen capacity during the contract period, in other terms that $q_{0}=A$. Indeed, suppose that for some $t$ in $[0, T]$, $q_{t}<A$, which means that $Q\left(p_{t}\right)<A$. As $A \leq Q\left(\frac{\varepsilon c}{\varepsilon-1}\right)$, necessarily, $p_{t}>\frac{\varepsilon c}{\varepsilon-1}$. A reduction in $p_{t}$ is beneficial for the producer in the short run, because the current price will be brought closer to the monopoly price so that profit will increase, and it will have no detrimental effect in the long run, because investment will not be affected. Indeed, as long as capacity is not binding at time $t$, the current price has no other effect than on current consumption. As a consequence, any contract price profile offered by the producer is necessarily such that the buyer will consume $A$ for all $t$ in $[0, T]$.

Step 3. Finally, we prove that the seller can never be worse off by restricting himself to a constant contract price. Since $\left\{p_{t}\right\}_{0 \leq t \leq T}$ is such that $q_{t}=A$ for all $t$ in $[0, T]$, any profile $\left\{p_{t}^{\prime}\right\}_{0 \leq t \leq T}$ included in the convex hull of $\left\{p_{t} \mid t \in I\right\}$, and such that

$$
\int_{0}^{T} p_{t}^{\prime} e^{-r t} d t=\int_{0}^{T} p_{t} e^{-r t} d t
$$

will produce the same choice $A$ by the buyer and the same consumption at each date.

In effect, the buyer's first-order condition w.r.t. $A$ is

$$
\int_{0}^{T}\left(u^{\prime}(A)-p_{t}\right) e^{-r t} d t+\frac{d}{d A} \int_{T}^{+\infty}(u(A)-p(A) A) e^{-r t} d t-k=0,
$$

which is also true if $\left\{p_{t}^{\prime}\right\}_{0 \leq t \leq T}$ is used instead of $\left\{p_{t}\right\}_{0 \leq t \leq T}$. Thus the producer's profit would be the same if he had set the following constant price:

$$
p_{0}=\frac{\int_{0}^{T} p_{t} e^{-r t} d t}{\int_{0}^{T} e^{-r t} d t} .
$$

To conclude, the largest possible profit can always be achieved with a constant price.

\section{A.3 Proof of Lemma 2}

The proof of this lemma is derived from Lemma 1's.

1. From Step $1, A \leq Q\left(\frac{\varepsilon c}{\varepsilon-1}\right)$. We know that $p_{T}=\max \left(\frac{\varepsilon c}{\varepsilon-1}, P(A)\right)$. Therefore $p_{T}=P(A) \geq \frac{\varepsilon c}{\varepsilon-1}$. Finally, since the buyer is active, the last inequality is strict.

2. Let's denote $Q\left(\frac{\varepsilon c}{\varepsilon-1}\right)$ by $\bar{A}$.

If the seller is passive, then by definition, $A=\bar{A}$ and $p_{T}=\frac{\varepsilon c}{\varepsilon-1}$. We reason by contradiction by assuming that $p_{0}<p_{T}$ (which implies in turn that $q_{0}=A$ ).

Given that $A=\bar{A}$, the maximum $p_{0}$ such that the buyer doesn't want to reduce his investment is defined by the first-order condition

$$
\int_{0}^{T}\left(u^{\prime}(A)-p_{0}\right) e^{-r t} d t+\frac{d}{d A} \int_{T}^{+\infty}(u(A)-p(A) A) e^{-r t} d t-k=0
$$


evaluated for $A=\bar{A}$.

This maximum price is denoted by $p_{0}^{*}$. Its expression is much simplified by using two facts: first, $u^{\prime}(\bar{A})=p(\bar{A})=\frac{\varepsilon c}{\varepsilon-1}$; second, $p^{\prime}(\bar{A}) A=-p(\bar{A}) / \varepsilon$. Equation (29) gives

$$
p_{0}^{*}=\frac{\varepsilon c}{\varepsilon-1}+\frac{e^{-r t}}{\varepsilon\left(1-e^{-r t}\right)}\left(\frac{\varepsilon c}{\varepsilon-1}-e^{r T} r \varepsilon k\right) .
$$

Clearly, if $p_{0}<p_{0}^{*}$, then $p_{0}$ is not optimal for the seller: increasing it will not decrease investment $A$ (thus second-period profits are preserved) and first-period consumption stays at $\bar{A}$ with a higher price (profits are increased). So we have proved that $p_{0} \geq p_{0}^{*}$ for the rest of the proof.

If $p_{0}^{*} \geq \frac{\varepsilon c}{\varepsilon-1}$, then $p_{0} \geq \frac{\varepsilon c}{\varepsilon-1}$, which we excluded by assumption.

If $p_{0}^{*}<\frac{\varepsilon c}{\varepsilon-1}$, we have to show that the seller wants a higher $p_{0}$ than $p_{0}^{*}$ (and not just stay at $p_{0}=p_{0}^{*}$ ), which implies that $A$ will not stay at $\bar{A}$.

When $p_{0}$ passes from $p_{0}^{*}$ to $p_{0}^{*}+d p_{0}$, given that $A$ will exhibit a first-order variation (it diminishes), the variation of the second-period profit is of second order (we had a repetition of the static maximum profit). To reach a conclusion, all we need is to evaluate the variation of profit for the first period. It is proportional to

$$
\bar{A}+\left(p_{0}^{*}-c\right) \frac{d A}{d p_{0}},
$$

for $A=\bar{A}$ and $p_{0}=p_{0}^{*}$.

The implicit definition of $A\left(p_{0}\right)$ is given by (29). After rearrangement of the terms, this gives

$$
p(A)\left[1+\frac{1}{\varepsilon} \frac{e^{-r T}}{1-e^{-r T}}\right]-\frac{r k}{1-e^{-r T}}=p_{0} .
$$

We can use again $u^{\prime}(A)=p(A)=\frac{\varepsilon c}{\varepsilon-1}$ and $p^{\prime}(A) A=-p(A) / \varepsilon$ on this implicit definition of $A\left(p_{0}\right)$ to find $\frac{d A}{d p_{0}}$. This yields

$$
\frac{d A}{d p_{0}}=\frac{1}{p^{\prime}(A)\left[1+\frac{1}{\varepsilon} \frac{e^{-r T}}{1-e^{-r T}}\right]} .
$$

The sign of (31) can now be computed: it is also the sign of

$$
p_{0}^{*}(1-\varepsilon)+\frac{r k}{1-e^{-r T}}+\varepsilon c .
$$

Clearly, it is positive if $p_{0}^{*}<\frac{\varepsilon c}{\varepsilon-1}+\frac{r k}{1-e^{-r T}}$, which is the case we are in. Therefore the seller doesn't want to choose $p_{0}=p_{0}^{*}$. We have the contradiction proving that $p_{0} \geq p_{T}=\frac{\varepsilon c}{\varepsilon-1}$.

The case $p_{0}>\frac{\varepsilon c}{\varepsilon-1}$ can be excluded since $p_{0}$ would be uselessly to large: lowering it to $\frac{\varepsilon c}{\varepsilon-1}$ would not decrease investment and would allow the seller to cash in the monopoly surplus all over the life of the investment. 
3. When the seller is passive, by definition $A=Q\left(p_{0}\right)$. When the buyer is passive, from Lemma 2 (point 2), $A=Q\left(p_{0}\right)$. When both the buyer and the seller are active, $A=Q\left(p_{T}\right) \neq Q\left(p_{0}\right)$. We will now prove by contradiction that $A>Q\left(p_{0}\right)$ cannot be an equilibrium. Indeed, it implies $p_{0}>p_{T}>\frac{\varepsilon c}{\varepsilon-1}$. But it is clearly profitable for the buyer to reduce $p_{0}$ : in the first period where $q_{0}=Q\left(p_{0}\right)$ he would get closer to his static monopoly profit, and in addition, this would induce the buyer to invest more, bringing second-period consumption closer to the seller's preferred level $\bar{A}$. Therefore, in an equilibrium with active buyer and active seller, necessarily $A<Q\left(p_{0}\right)$.

\section{A.4 Proof of Theorem 1}

Whenever the buyer is active, he solves:

$$
\max _{A} \frac{1-e^{-r T}}{r}\left(u(A)-p_{0} A\right)+\frac{e^{-r T}}{r}(u(A)-P(A) A)-k A,
$$

where the first term is the present buyer utility from the contract, the second term is the present utility for the subsequent period, and the third is investment cost. The fact that the buyer is active enables us to replace $p_{T}$ with $P(A)$; Lemma 2 (1.) enables us to replace consumption in the first period with $A$.

We find

$$
A=Q\left(\frac{p_{0}+\frac{r}{1-e^{-r T}} k}{1+\frac{1}{\varepsilon} \frac{e^{-r T}}{1-e^{-r T}}}\right)
$$

Since the seller is active, $p_{0}<p_{T}$ : the seller offers a lower price in the first period so that the buyer is incited to invest more, then once the capacity is fixed he sets a higher price. To choose $p_{0}$, the seller solves

$$
\max _{p_{0}}\left[\frac{1-e^{-r T}}{r}\left(p_{0}-c\right)+\frac{e^{-r T}}{r}\left(\frac{p_{0}+\frac{r}{1-e^{-r T}} k}{1+\frac{1}{\varepsilon} \frac{e^{-r T}}{1-e^{-r T}}}-c\right)\right] Q\left(\frac{p_{0}+\frac{r}{1-e^{-r T}} k}{1+\frac{1}{\varepsilon} \frac{e^{-r T}}{1-e^{-r T}}}\right) .
$$

This yields the optimal contract price

$$
p_{0}=\frac{1}{1-e^{-r T}}\left[\left(1-\frac{\varepsilon e^{-r T}}{\varepsilon+e^{-r T}}\right) \frac{\varepsilon c}{\varepsilon-1}+\left(1-\frac{\varepsilon^{2} e^{-r T}}{\varepsilon+e^{-r T}}\right) \frac{r k}{\varepsilon-1}\right] .
$$

Finally, we need to check under which conditions the buyer and the seller are actually active. To ensure $A<Q\left(\frac{\varepsilon c}{\varepsilon-1}\right)$ and $A<Q\left(p_{0}\right)$, we calculate from equations (36) and (38) that the ratio of the production and investment costs must verify respectively $\frac{c}{r k}<\varepsilon e^{r T}$ and $\frac{c}{r k}<(\varepsilon-1) e^{r T}-\frac{1}{\varepsilon}$. Clearly the latter inequality implies the former: both players are active if and only if

$$
\frac{c}{r k}<(\varepsilon-1) e^{r T}-\frac{1}{\varepsilon}
$$


To complete the proof of (c) in the theorem, we use equation (36),

$$
p_{T}=\frac{p_{0}+\frac{r}{1-e^{-r T}} k}{1+\frac{1}{\varepsilon} \frac{e^{-r T}}{1-e^{-r T}}} .
$$

For case (b), we have both $p_{0}=p_{T}$ and equation (40). Thus the price is $r e^{r T} \varepsilon k$. From Lemma 2 (1. and 2.), we can deduce that a necessary and sufficient condition for the buyer to be active is $p_{T}>\frac{\varepsilon c}{\varepsilon-1}$.

Case (a) remains to be proved. When the buyer is passive, he knows that the seller will set $p_{T}=\frac{\varepsilon c}{\varepsilon-1}$. The price prevailing after contract expiry is

$$
p_{T}=\min \left\{\frac{p_{0}+\frac{r}{1-e^{-r T}} k}{1+\frac{1}{\varepsilon} \frac{e^{-r T}}{1-e^{-r T}}}, \frac{\varepsilon c}{\varepsilon-1}\right\} .
$$

This enables us to write the following necessary and sufficient condition for having a passive buyer:

$$
p_{0}-\frac{\varepsilon c}{\varepsilon-1}<\frac{c-(\varepsilon-1) e^{r T} r k}{(\varepsilon-1)\left(e^{r T}-1\right)}
$$

Since this situation is characterized by $p_{0}=\frac{\varepsilon c}{\varepsilon-1}$, the left hand-side is equal to zero, and the following result obtains: when

$$
\frac{c}{r k} \geq(\varepsilon-1) e^{r T}
$$

the buyer is passive - and so is the seller.

QED.

\section{A.5 Comparative statics w.r.t. contract duration: a complement}

The case where $\frac{c}{r k}<\varepsilon-1-\frac{1}{\varepsilon}$ was detailed in subsection 4.1. Now assume the opposite holds. Let

$$
\left\{\begin{aligned}
T_{\text {inf }} & =\frac{1}{r} \ln \left(\frac{c}{(\varepsilon-1) r k}\right), \\
T_{\text {sup }} & =\frac{1}{r} \ln \left(\frac{\varepsilon c+r k}{\varepsilon(\varepsilon-1) r k}\right) .
\end{aligned}\right.
$$

The three cases mentioned in Theorem 1 correspond respectively to $T \leq T_{\text {inf }}, T_{\text {inf }}<$ $T \leq T_{\text {sup }}$ and $T>T_{\text {sup }}$, and to zones (a), (b), and (C) in Figure 1.

By assumption, $T_{\text {sup }}>0 ; T_{\text {inf }}$ is positive when $\frac{c}{r k}>\varepsilon-1$. In what follows, we assume this is also true (else Case (a) is simply empty).

For the buyer, being active, i.e. reducing $A$ below the monopoly quantity, implies an immediate benefit (lower investment expenses), and from $T$ on, a cost (higher $p_{T}$ ).

For the seller, being active, i.e. reducing $p_{0}$ below the marginal willingness to pay of the buyer during the contract, implies an immediate cost (lower first-stage profits), and from $T$ on, a benefit (larger volumes due to increased investment). 
(a) $T \leq T_{\mathrm{inf}}$ : both parties are passive. For the buyer, the cost of being active is coming up too early, and he prefers to stay passive and invest $A=Q\left(\frac{\varepsilon c}{\varepsilon-1}\right)$.

(b) $T_{\text {inf }}<T \leq T_{\text {sup }}$ : only the buyer is active. The cost of being active is delayed, it is worth reducing $A$ : equalizing marginal cost and marginal benefit $\left(k=\frac{e^{-r T}}{r} \frac{P(A)}{\varepsilon}\right)$ yields $A=Q\left(r e^{r T} \varepsilon k\right)$.

(C) $T>T_{\text {sup }}$ : both parties are active. When $T$ becomes large, capacity risks to decrease too much if the seller stays passive. He becomes active and offers a lower $p_{0}$ to stimulate investment. Decreasing $p_{0}$ is a good strategy as long as $T$ is not too large. But as the contract length approaches infinity, the hold-up period becomes too remote: the seller seeks to preserve profits made during the contract by increasing $p_{0}$.

See Figure 1.

\section{A.6 Comparative statics w.r.t. investment cost}

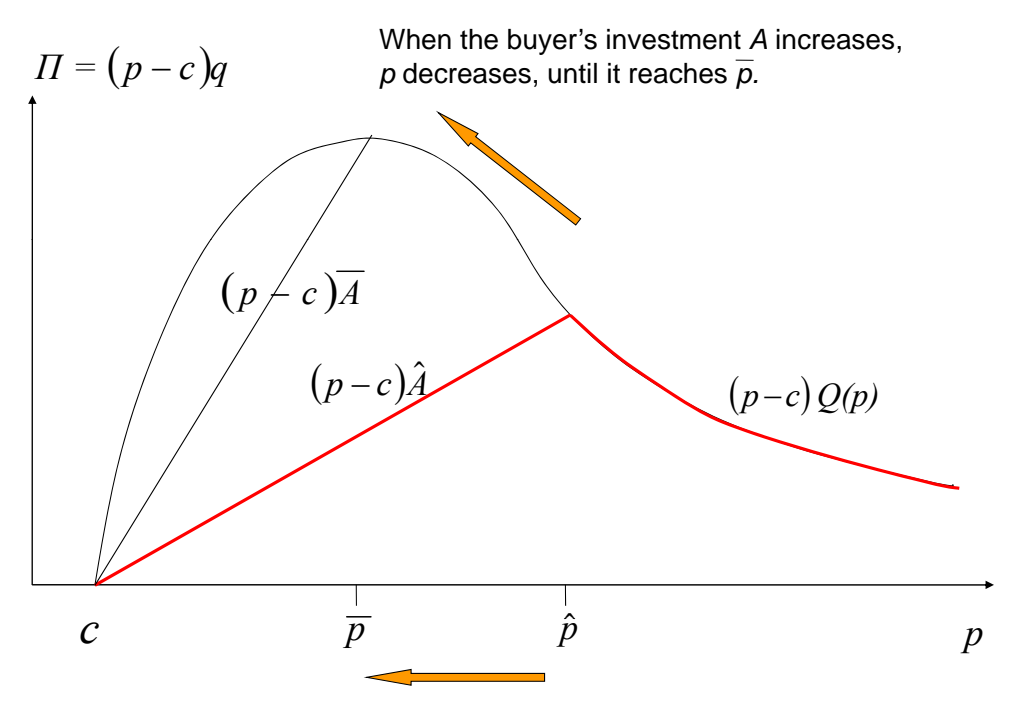

Figure 4: Equilibrium prices as a function of the investment cost.

Let

$$
\left\{\begin{aligned}
k_{\text {inf }} & =\frac{e^{-r T}}{r} \frac{c}{\varepsilon-1}, \\
k_{\text {sup }} & =\frac{e^{-r T}}{r} \frac{c}{\varepsilon-1-\frac{1}{\varepsilon} e^{-r T}} .
\end{aligned}\right.
$$

For all $T, 0<k_{\text {inf }}<k_{\text {sup }}$. The three cases mentioned in Theorem 1 correspond respectively to $k \leq k_{\text {inf }}, k_{\text {inf }}<k \leq k_{\text {sup }}$ and $k>k_{\text {sup }}$, and and to zones (a), (b), and (c) in Figure 4. 
(a) $k \leq k_{\text {inf }}$ : both parties are passive. In the second stage, the seller will be able to set his unconstrained monopoly price $p_{T}=\frac{\varepsilon c}{\varepsilon-1}$ only if the invested capacity is sufficiently large. Therefore, as long as setting his monopoly price $p_{0}=\frac{\varepsilon c}{\varepsilon-1}$ in the first stage induces a sufficiently large investment $A \geq Q\left(\frac{\varepsilon c}{\varepsilon-1}\right)$, the seller has no incentive to deviate, and he will set in both stages $p_{0}=p_{T}=\frac{\varepsilon c}{\varepsilon-1}$. This will be the case when the investment cost $k$ is not too large: the buyer responds passively to the contract price by setting $A=Q\left(\frac{\varepsilon c}{\varepsilon-1}\right)$.

When $k$ becomes larger, the buyer compensates the higher unit cost of equipment by reducing his investment to $A<Q\left(\frac{\varepsilon c}{\varepsilon-1}\right)$. The seller then necessarily sets in the second stage the corresponding hold-up price $p_{T}=P(A)>\frac{\varepsilon c}{\varepsilon-1}$. In the first stage, two possible choices are available to the seller, knowing that $q_{0}=\min \left\{A, Q\left(p_{0}\right)\right\}$. Either he passively sets $p_{0}$ such that at equilibrium $p_{0}=p_{T}=P(A)$ and in particular $p_{0}=P\left(q_{0}\right)$ (case (b) below), or he strategically sets the contract price below the marginal willingness to pay of the buyer $\left(p_{0}<P\left(q_{0}\right)\right)$ : he forgoes first-stage profits in order to induce the buyer to invest in a larger equipment capacity, thus preserving second-stage profits (case (C) below).

(b) $k_{\text {inf }}<k \leq k_{\text {sup }}$ : only the buyer is active. For intermediate values of $k$, the investment level is still close to the seller's preferred level $Q\left(\frac{\varepsilon c}{\varepsilon-1}\right)$, therefore setting $p_{0}=$ $p_{T}>\frac{\varepsilon c}{\varepsilon-1}$ yields only a second-order loss in both stages compared to the initial situation with a low $k$, whereas setting $p_{0}<P(A)=p_{T}$ would cause a first-order loss $\left(P(A)-p_{0}\right) A$.

(C) $k>k_{\text {sup }}$ : both parties are active. When $k$ becomes sufficiently large, setting $p_{0}<p_{T}$ in order to increase $A$ becomes worthwhile, since the positive volume effect in both stages offsets the first-stage loss. In this case only, the contract price and the hold-up price differ. The contract price can even be a decreasing function of $k$, and become negative when $k$ is high, if the contract length is short enough: the seller accepts a loss that will be compensated by larger sales volumes after contract expiry.

\section{A.7 Proof of Proposition 2}

Proof in the text.

\section{A.8 Proof of Proposition 3}

Profit of the seller. The seller's profit can be expressed as a function of the contract duration:
(a) $T \leq T_{\text {inf }}$ :
$\Pi(T)=\frac{1}{r} \frac{c}{\varepsilon-1} Q\left(\frac{\varepsilon c}{\varepsilon-1}\right) ;$
(b) $T_{\text {inf }}<T \leq T_{\text {sup }}$ :
$\Pi(T)=\frac{1}{r}\left(r e^{r T} \varepsilon k-c\right) Q\left(r e^{r T} \varepsilon k\right) ;$
(c) $T>T_{\text {sup }}$ :
$\Pi(T)=\frac{1}{r} \frac{c+r k}{\varepsilon-1} Q\left(\frac{\varepsilon^{2}(c+r k)}{\left(e^{-r T}+\varepsilon\right)(\varepsilon-1)}\right)$.

The seller's profit is a non-increasing function of the contract duration: he always prefers shorter contracts, but actually any contract duration between 0 and $T_{\text {inf }}$ is equivalent for 
him.

\section{Surplus of the buyer.}
(a) $T \leq T_{\mathrm{inf}}$ :
$S(T)=\frac{1}{r}\left(\frac{\varepsilon c}{(\varepsilon-1)^{2}}-r k\right) Q\left(\frac{\varepsilon c}{\varepsilon-1}\right) ;$
(b) $T_{\text {inf }}<T \leq T_{\text {sup }}$ :
$S(T)=\frac{1}{r} \frac{1-e^{-r T}+\frac{1}{\varepsilon} e^{-r T}}{\varepsilon-1} r e^{r T} \varepsilon k Q\left(r e^{r T} \varepsilon k\right) ;$
(c) $T>T_{\text {sup }}$ :
$S(T)=\frac{1}{r} \frac{1-e^{-r T}+\frac{1}{\varepsilon} e^{-r T}}{\varepsilon-1} \frac{\varepsilon^{2}(c+r k)}{\left(e^{-r T}+\varepsilon\right)(\varepsilon-1)} Q\left(\frac{\varepsilon^{2}(c+r k)}{\left(e^{-r T}+\varepsilon\right)(\varepsilon-1)}\right)$.

The buyer's surplus function is not monotonous with respect to $T$ : it is first constant, then decreasing, then increasing and it tends to a finite limit. In addition, the thresholds in $T$ are not always positive, so that depending on the value of the parameters, cases (a) and (b) can be empty.

First suppose $\frac{c}{r k} \leq \varepsilon-1-\frac{1}{\varepsilon}$ : only case (C) exists, and since the surplus is a strictly increasing function of $T$ in this case, the buyer always prefers the longest possible contract.

Now if $\varepsilon-1-\frac{1}{\varepsilon} \leq \frac{c}{r k} \leq \varepsilon-1$, we are either in case (b) or in case (C), so the buyer's surplus is highest either for $T=0$, or when $T \rightarrow+\infty$. Comparing the two values yields the following result: the buyer's surplus is highest for $T \rightarrow 0$ if and only if

$$
\frac{c}{r k} \leq(\varepsilon-1) \varepsilon^{\frac{1}{\varepsilon-1}}-1 .
$$

Finally, when $\frac{c}{r k} \geq \varepsilon-1$, all three cases exist. Clearly the buyer's surplus is maximal either for $T \rightarrow 0$, or when $T \rightarrow+\infty$. Let $y=\frac{c}{r k}$. After rearrangement, we find that the buyer prefers the shortest possible contract whenever

$$
\left(1-\frac{(\varepsilon-1)^{2}}{\varepsilon y}\right)\left(1+\frac{1}{y}\right)^{\varepsilon-1} \geq 1 .
$$

The derivative with respect to $y$ of the left hand-side has a unique root $\varepsilon(\varepsilon-1)$. The LHS is increasing then decreasing, it tends to $-\infty$ when $y$ tends to zero, and to 1 when $y$ tends to $+\infty$, thus it is equal to 1 for a unique value of $y$. Therefore there is a unique $y^{*}$ such that the buyer's surplus is maximal when $T \rightarrow+\infty$ whenever $y \leq y^{*}$, and it is maximal for $T \rightarrow 0$ whenever $y \geq y^{*}$.

In addition, the LHS is higher than 1 for $y=\varepsilon(\varepsilon-1)$, which means that $0<y^{*}<$ $\varepsilon(\varepsilon-1)$. As a consequence, when the elasticity parameter $\varepsilon$ is close to 1 , the threshold $y^{*}$ is close to zero, so that for all values of the ratio $\frac{c}{r k}$ that are bounded away from zero, the buyer prefers the shortest possible contract.

\section{A.9 Proof of Proposition 4}

The model is solved by backward induction.

Choice of $q$ and $p$. From Lemma 2 (1. and 2.), $q=Q(p)$ with $p=\max \left\{\frac{\varepsilon c}{\varepsilon-1}, P(A)\right\}$. 
Choice of $A$. Solving backwards, we come to the investment choice of the buyer. See Figure 5. In the "active-buyer" regime the price $P(A)$ decreases with respect to $A$. Thus as long as this regime prevails, a larger investment yields a bigger price reduction. But when the investment attains the monopoly quantity, this effect stops because the seller would never decrease the price below the monopoly price $\frac{\varepsilon c}{\varepsilon-1}$ : it is never worth investing more than $\bar{A}=Q\left(\frac{\varepsilon c}{\varepsilon-1}\right)$. Actually, when the investment cost is large, the buyer prefers to invest less.

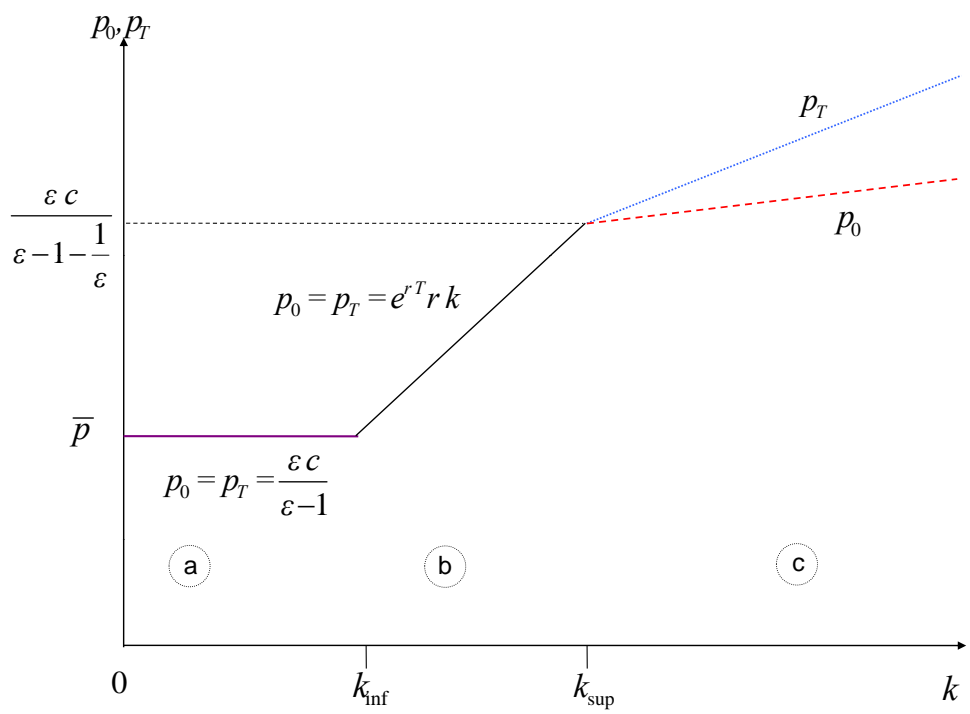

Figure 5: Impact of the investment choice on the price in model $A p$ with $\bar{p}=\frac{\varepsilon c}{\varepsilon-1}$.

Thus, the buyer's surplus-maximization program can be expressed as

$$
\begin{aligned}
& \max _{A} S(A)=\frac{1}{r}(u(A)-(P(A)+r k) A) \\
& \text { s.t. } A \leq Q\left(\frac{\varepsilon c}{\varepsilon-1}\right) .
\end{aligned}
$$

The solution $A$ is interior when the active-buyer regime prevails: the seller adjusts his price so that $q=A$. Simple calculations yield

$$
p=\varepsilon r k \text {. }
$$

This situation combines the features of monopoly and monopsony. In the regime where $p=P(A)$, the buyer chooses $A$ as a monopsonist to maximize $S(A)=\frac{1}{r}(u(A)-P(A) A-r k A)$. The first-order condition can be rewritten $\frac{P(A)+r k-u^{\prime}(A)}{P(A)}=\frac{-P^{\prime}(A) A}{P(A)} \equiv \frac{1}{\varepsilon}$. However, while in the monopsony case the price equals the marginal cost, here the seller's market power 
allows him to set a price equal to the marginal utility of the buyer $\left(P(A)=u^{\prime}(A)\right)$. Thus the equation can be rewritten $p(A)=\varepsilon r k$.

The seller is constrained by this investment choice if he cannot set his monopoly price $\frac{\varepsilon c}{\varepsilon-1}$ because $Q\left(\frac{\varepsilon c}{\varepsilon-1}\right)>Q(\varepsilon r k)$, or equivalently, $\frac{\varepsilon c}{\varepsilon-1}<\varepsilon r k$. This is the case when $\frac{c}{r k}<\varepsilon-1$.

Conversely, when $\frac{c}{r k}>\varepsilon-1$ then $\varepsilon r k<\frac{\varepsilon c}{\varepsilon-1}$, thus investing $Q(\varepsilon r k)$ will not prevent the seller from setting his monopoly price. The buyer would do better to invest passively $Q\left(\frac{\varepsilon c}{\varepsilon-1}\right)$. 